\title{
Thermoregulation of plant systems as a factor of production processes
}

\author{
Alexander Grishin, Andrey Grishin, Inna Knyazeva*, and Vladimir Grishin \\ Federal Scientific Agroengineering Center VIM,109428, Russia
}

\begin{abstract}
Thermoregulation in plant systems is one of the main functions of transpiration, which plays the role of the upper terminal mover of the water current. It creates a continuous flow of water from the root system to the leaves and other vegetative organs of the plant, linking them into a single whole. This flow transports dissolved mineral and partially organic nutrients. The study of thermoregulation on plants of the strawberry garden variety Asia was conducted in 2019-2020 in the department of Closed Artificial Agroecosystems for Plant Growing at the Federal Scientific Agroengineering Center VIM, Moscow. The object of the study was thermoregulation processes occurring in plant systems. The subject of the study was the culture of garden strawberry Fragaria $\times$ ananassa (Weston.) Duchesne of the 'Asia' variety. As a source of light energy (LE) we used a thirty-six-watt bispectral lamp, consisting of 12 three-watt LEDs of red (660 $\mathrm{nm})$ and blue $(450 \mathrm{~nm})$ colors at a ratio of 10:2, providing constant illumination of the culture. The study results allowed for a digital control of the efficiency of production processes in plant systems, due to the implementation of a step-by-step control algorithm in digital devices. The algorithm makes it possible to establish the maximum temperature difference between the leaf surface and the air.
\end{abstract}

\section{Introduction}

Thermoregulation in plant systems is one of the main functions of transpiration, which plays the role of the upper terminal mover of the water current. It creates a continuous flow of water from the root system to the leaves and other vegetative organs of the plant, linking them into a single whole. This flow transports dissolved mineral and partially organic nutrients [1]. Transpiration is a complex biological phenomenon associated with the operation of stomata. These pores facilitate evaporation creating a gradient of water flow through the plant [2-5]. As a result, this phenomenon is the most important means of protecting leaves from overheating, which is very important for the life of the plant organism, and primarily for the processes of photosynthesis. Evaporation of water, like any other liquid, from the surface occurs due to the kinetic energy and constant motion of particles. A number of authors [6-8]

\footnotetext{
* Corresponding author: knyazewa.inna@yandex.ru
} 
considered the process of thermoregulation from the standpoint of thermodynamics as four successive stages:

Stage 1. Heating the thermal component of light energy (LE) coming from the light source, and the evaporation of water by the stomata of the leaf - El: Ev = Es - Ee, where Ev is the amount of heat transferred from water to the vapour-gas medium of air; Ee is the heat of evaporation;

Stage 2. Cooling of plants until tv=tl, (where tv is the temperature of the vapour-gas medium; tl is the temperature of the leaf stomata) equality is established, heat transfer to the vapour-gas medium will stop $(\mathrm{Ep}=0)$ and all the heat supplied from the stomata to the interface will be spent on evaporation, that is, $\mathrm{El}=\mathrm{Ee}$;

Stage 3. In the case of the earlier specified equality the pressure at the interface remains higher than in air, subsequently, evaporative cooling does not stop and tv becomes lower than tl and tair.

Stage 4. As a result of a decrease in ts, the equality $t v=t l$ is achieved and the heat flow from the liquid stops whereas the heat flow from the gaseous medium Eair becomes equal to Ee.

Subsequently, liquid is evaporated at a constant temperature, which is based on stationary evaporation in an open nonlinear dynamic thermoregulatory cooling system. The stationarity of a random process is characterized by the constancy of the expectation and variance. In other words, the temperature of the leaf plate is stabilized, that is, thermoregulation plays the role of a self-organizing process of evaporative cooling (SPEC), proceeding as evaporation $[9,12,13]$. In this case, as can be seen from the description of the stages, there is an energy exchange between the external environment, which has a source of the thermal component of LE, and the plant system (Fig. 1) [10].

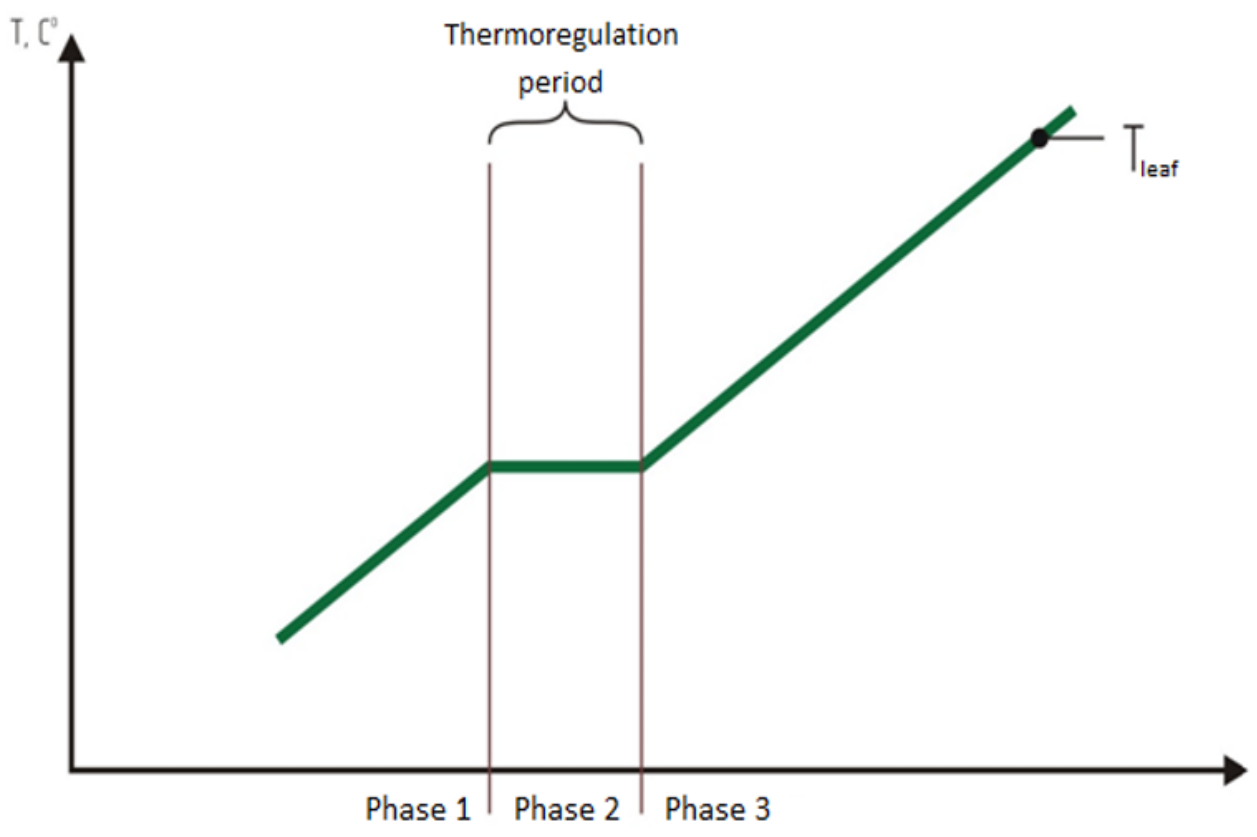

Fig. 1. Leaf temperature with increasing air temperature (phase 1 - ambient temperature is below optimal; phase 2 - ambient temperature is higher than optimal; stomatal apparatus is functioning and the plant starts the cooling process (thermoregulation); phase 3 - ambient temperature is higher than optimal; the plant is not able to start the cooling process due to the limiting opening of the stomata 
Thermodynamic processes also have another, bioenergetic content, since they are based on synergistic relationships that link the energetics of photosynthesis with the energetics of evaporative cooling, which ensures the optimum temperature of photosynthesis, thereby regulating mineral nutrition in general, due to the flow of nutrients through the plant. These two bioenergetic processes determine the productivity of plant systems [11, 15].

The effectiveness of mineral nutrition depends on the amount of nutrients entering the plant system, which, in turn, depends on the intensity or consumption of the nutrient solution through the plant. The consumption is determined according to the SPEC [11]. The amount of cooling water in plants for a certain period of time is determined by the expression:

$$
q_{e}=\frac{\left(t_{l}-t_{o}\right) M_{l} C_{l}}{r_{w}}
$$

where $\mathrm{Ml}$ is the total mass of leaves; $\mathrm{Cl}$ is the specific heat of the mass of leaves; $\mathrm{rw}$ is the specific heat of water evaporation; $t$ is the leaf temperature of the plant; to is the temperature optimum of photosynthesis.

The physical meaning of the expression is that the thermal component of the LE energy, which depends on the difference between the temperature of the heated leaf and stabilization temperature, cools the leaf due to the SPEC. The intensity of the mineral nutrition consumption through the plant has a maximum value at the end of phase 2 , since the air temperature continues to rise, while the period of stabilization of the leaf temperature (thermoregulation) is completed and the leaf surface heats simultaneously with the air while the maximum difference between them is preserved. The maximum flow rate at the end of phase 2 corresponds with a maximum photosynthesis rate, since to was reached $[5,13]$.

Thus, the process of thermoregulation, which links the energetics of photosynthesis and the energetics of evaporative cooling, contributes to the control of nutrient consumption through plant tissues [14].

The purpose of the study was to experimentally confirm the role of thermoregulation as a factor of production processes in a plant organism.

\section{Materials and methods}

The process of thermoregulation on plants of the Asia strawberry garden variety was studied in 2019-2020 in the department of Closed Artificial Agroecosystems for Plant Growing at the Federal Scientific Agroengineering Center VIM, Moscow. The object of the study was thermoregulation processes occurring in plant systems. The subject of the study was the garden strawberry culture Fragaria ×ananassa (Weston.) Duchesne of the Asia variety.

Strawberry plants were grown in a container with a soil substrate and an automatic moisture maintenance system. The container with the substrate had a film coating to exclude evaporation from the substrate surface.

As a source of light energy (LE) we used a thirty-six-watt bispectral lamp, consisting of 12 three-watt LEDs of red $(660 \mathrm{~nm})$ and blue $(450 \mathrm{~nm})$ colors at a ratio of 10:2, providing constant illumination of the culture.

Despite the constancy of illumination and stabilization of the temperature of the leaf plate due to thermoregulatory evaporation, heating had random deviations due to errors in the parameters of the external environment (air movement, plant growth, and its approach to the light source) [5-7]. These deviations were checked for randomness from the expectation using the statistical method of successive differences. 
The leaf temperature was measured using a KELVIN IKS 4-20/5 non-contact thermometer; the measurement error was $0.5^{\circ} \mathrm{C}$.

The characteristics of the processes were measured thrice using an automatic recorder within 19 hours. The obtained experimental data were processed by the methods of mathematical statistics using Microsoft Office Excel 2016 and STADIA 8.0.

\section{Results and discussion}

The purpose of the experiment presented in Table 1 and Figure 2 was to study thermoregulation processes. The results of the experiment show that a period of thermoregulation lies within the time interval of 5-10 hours. We also registered some random deviations. This was confirmed by the calculation for randomness $\tau_{\mathrm{f}}>\tau_{\mathrm{t}}$, which corresponded to the statistical calculations of random deviations of the $\mathrm{T}_{\text {lef }}$ sample. In the interval of 5-10 minutes the leaf temperature remained stable at $22.4^{\circ} \mathrm{C}$.

Table 1 Average experimental values and statistical calculation of testing the hypothesis about the randomness of deviations in the sample of the leaf temperature stabilization period from the expectation

\begin{tabular}{|c|c|c|c|c|c|c|c|}
\hline $\begin{array}{l}\text { Time, } \\
\text { hour }\end{array}$ & $\begin{array}{l}\mathrm{T}_{\text {air }}, \\
{ }^{\circ} \mathrm{C}\end{array}$ & $\begin{array}{l}\mathrm{T}_{\text {leaf }}, \\
{ }^{\circ} \mathrm{C}\end{array}$ & $\mathrm{Ta}-\mathrm{Tl}$ & & & & \\
\hline 1 & 21.09 & 20.15 & 0.94 & & & & \\
\hline 2 & 21,7 & 21.11 & 0.59 & & & & \\
\hline 3 & 21.93 & 21.44 & 0.49 & & & & \\
\hline 4 & 21.98 & 21.77 & 0.21 & & $\mathrm{x}_{\mathrm{n}}\left(\mathrm{T}_{\mathrm{l}}\right)$ & $a=x_{n}-x_{n}-1$ & $\Sigma \mathrm{a}^{2}$ \\
\hline 5 & 22.34 & 22.03 & 0.31 & & 22.03 & 0.26 & 0.0676 \\
\hline 6 & 23.08 & 22.29 & 0.79 & & 22.29 & 0.14 & 0.0196 \\
\hline 7 & 23.16 & 22.43 & 0.73 & & 22.43 & 0.14 & 0.0196 \\
\hline 8 & 23.21 & 22.57 & 0.64 & & 22.57 & -0.04 & 0.0016 \\
\hline 9 & 23.34 & 22.53 & 0.81 & & 22.53 & 0.04 & 0.0016 \\
\hline 10 & 23.39 & 22.57 & 0.82 & & 22.57 & & 0 \\
\hline 11 & 23.42 & 23.01 & 0.41 & Mean & 22.40333 & $\Sigma \mathrm{a}^{2}$ & 0.0424 \\
\hline 12 & 23.42 & 22.9 & 0.52 & $\begin{array}{l}\text { Mean } \\
\text { square } \\
\text { deviation, } \\
\sigma\end{array}$ & 0.211912 & $\mathrm{C}^{2}=\Sigma \mathrm{a}^{2} / 2(\mathrm{n}-1)$ & 0.01696 \\
\hline 13 & 23.44 & 23.06 & 0.38 & Disp, $\sigma 2$ & 0.044907 & $\tau_{\mathrm{f}}=\mathrm{C}^{2} / \sigma^{2}$ & 0.377672 \\
\hline 14 & 23.91 & 23.47 & 0.44 & & & $\begin{array}{l}\tau_{\mathrm{t}}=1-2.33 / \\
\sqrt{n+1}\end{array}$ & 0.119343 \\
\hline 15 & 24.52 & 24.09 & 0.43 & & & & \\
\hline 16 & 24.52 & 23.87 & 0.65 & & & & \\
\hline 17 & 24.54 & 24.03 & 0.51 & & & & \\
\hline 18 & 24.6 & 24.04 & 0.56 & & & & \\
\hline 19 & 25.62 & 25.07 & 0.55 & & & & \\
\hline
\end{tabular}

We also note that the 10 o'clock mark corresponds to the end of phase 2 and has the maximum value of the temperature difference $\mathrm{Tw}-\mathrm{Tl}$. According to the above formula, the amount of cooling water, due to the temperature difference, will correspond to the maximum intensity of the consumption of mineral nutrition. 


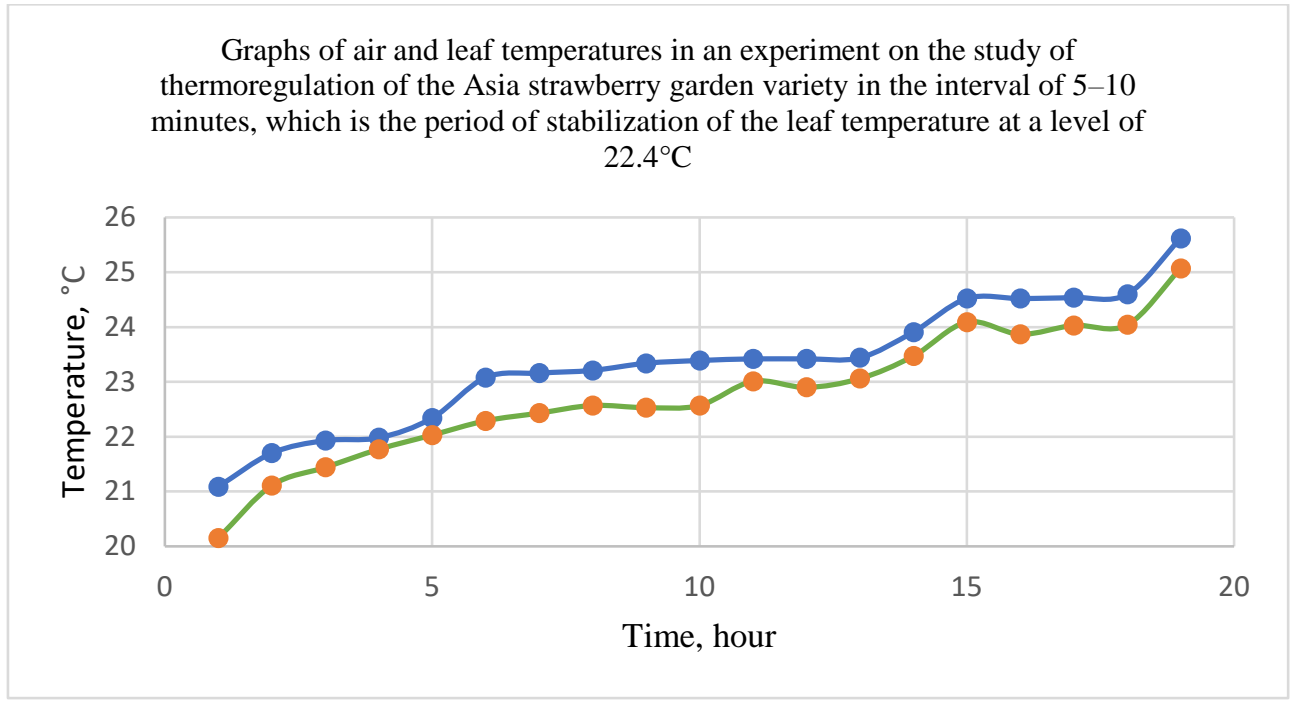

Fig. 2. Temperature of ambient air and leaf of plants of the 'Asia' variety

The study results make it possible to solve the issue of digital control of the efficiency of production processes in plant systems, due to the implementation of a step-by-step control algorithm in digital devices. Algorithm allows for the determination of the maximum temperature difference between the surface of the leaf and the air. When searching for the maximum temperature difference we compared current and maximum temperatures. If the current temperature difference is greater than the maximum, i.e. $\Delta$ Tcurrent $>\Delta \mathrm{Tmax}$, then the maximum value of the temperature difference has not yet been reached and the value of the current difference is assigned to it. If the temperature difference stops growing, i.e. $\Delta$ Tcurrent $\leq \Delta \mathrm{Tmax}$, it is considered that a transition from phase 2 to phase 3 was found and the ambient temperature at a given time is optimal for the highest moisture consumption by the plant. In this case, the ambient temperature is set equal to the current ambient temperature (Tset = Tcurrent). As a result, the temperature search mode is disabled and the set Tset is maintained.

\section{Conclusions}

1. Thermodynamic processes are characterized by a bioenergetic content, since they are based on synergistic relationships that link the energetics of photosynthesis with the energetics of evaporative cooling, which ensures the optimum temperature of photosynthesis, thereby regulating mineral nutrition in general, due to the flow of nutrients through the plant. These two bioenergetic processes determine the productivity of plant systems.

2. Thermoregulation by means of SPEC stabilizes the leaf temperature at the level of the maximum values of photosynthesis during a certain period of time.

3. The maximum temperature difference between the air and the leaf leads to the maximum consumption of nutrients through plant tissues.

4. The study results make it possible to solve the issue of digital control of the efficiency of production processes in plant systems, due to the implementation of a step-by-step control algorithm in digital devices. 


\section{References}

1. S.T. Michaletz, M.D. Weiser, J. Zhou, M. Kaspari, B.R. Helliker, B.J., Enquist Plant Thermoregulation: Energetics, Trait-Environment Interactions, and Carbon Economics, Trends in Ecology \& Evolution, 12(30), pp. 715-724 (2015) doi.org/10.1016/j.tree.2015.09.006

2. A. Barekova, V. Barek, M. Kovacova, B.-K. Novotna, Climate conditions impact on the sap flow into plants and their dendrometric changes, Journal of ecological engineering, 21(6), pp. 224-228 (2020) doi: 10.12911 / 22998993/124077.

3. A.Z. Venturin, C.M. Guimaraes, E. Fernandes de Sousa, J.A.M. Filho, R.P. Weverton, et al., Using a crop water stress index based on a sap flow method to estimate water status in conilon coffee plants, Agricultural water management, 241(106343), (2020) doi: 10.1016 / j.agwat.2020.106343.

4. Y. Andreev, T. Makeeva, E. Pukhova, V. Sevryugin, G. Sherstnev, Technical means of digital information processing systems Moscow, Ivan Fedorov Moscow State University of Printing Arts, 74 p (2015).

5. X. Kai, G. Liang, Y. Hong, A naturally optimized mass transfer process: The stomatal transpiration of plant leaves, Journal of Plant Physiology, 234 (235), p 138-144 (2019).

6. A.P. Grishin Energy-information technologies of irrigation, Technologies in agriculture, 2, p. 22-25 (2010).

7. A.A. Grishin, A.P. Grishin, Plant transpiration as a dissipative process with a temporal fractal structure, Nainovite scientific comprehension, Materials of 10th international scientific conference, 27, p. 44-51 (2014).

8. A.P. Grishin, Energy flows of the transpiration process in plants. Intelligent machine technologies and equipment for the implementation of the state program for the development of agriculture, Collection of scientific reports of the International Scientific and Technical Conference, p. 210-214 (2015)

9. V. Mudrik, I. Knyazeva, A. Kosobrukhov, T. Pigulevskaya, Changes in the photosynthetic characteristics of Plantago major plants caused by soil drought stress, Plant Growth Regulation, 40(1), p. 1-9 (2003).

10. V. Mudrik, P. Stoyanov, B.N. Ivanov, Evaluation of maize productivity considering solar energy use limitation by environmental factors, Photosynthesis Research, 66, p. 177 (2000).

11. H. Hermann, Synergetics, 405 p. (1980)

12. V. Karasev, M. Karaseva, D. Mukhortov, Diagnostics of the physiological state of coniferous trees by bioelectric and temperature indicators, Lesovedenie, 2, pp. 162174. (2020) doi: 10.31857 / S0024114820010088.

13. I. Sventitsky, Energy saving in the agro-industrial complex and energy extremity of self-organization, VIESH, 466 p. (2007).

14. K. Kensuke, Y. Daisuke, K. Kota, K. Masaharu, Spatiotemporal variability of leaf photosynthesis and its linkage with microclimates across an environment-controlled greenhouse, Biosystems Engineering, 195 (2020). p. 97-115

15. G. Nikolaou, D. Neocleous, E. Kitta, N. Katsoulas, Estimation of Aerodynamic and Canopy Resistances in a Mediterranean Greenhouse Based on Instantaneous Leaf Temperature Measurements. Agronomy 2020, 10(12) (1985) doi.org/10.3390/agronomy10121985 